Check for updates

Cite this: RSC Adv., 2019, 9, 30809

\title{
Pseudocapacitance electrode and asymmetric supercapacitor based on biomass juglone/activated carbon composites $\uparrow$
}

\author{
Xin He, ${ }^{a}$ Qian Chen, ${ }^{a}$ Xiling Mao, ${ }^{a}$ Weichen Liu, ${ }^{a}$ Yujiu Zhou, ${ }^{a}$ Wenyao Yang, ${ }^{b}$ \\ Yajie Yang $^{a}$ and Jianhua Xu (D)*a
}

\begin{abstract}
A novel electrode material incorporating renewable biomass-derived juglone biomolecules with commercial activated carbon (AC) granules has been through simple ultrasonic dispersion and dissolution-recrystallization and was found to exhibit good electrochemical performance. The juglone biomolecules are prepared by an ultrasound-assisted extraction method from abandoned walnut peel, which decreases pollution and increases economic efficiency. Through the dissolution-recrystallization process with $A C$, a hierarchical structure with nanosized juglone particles was obtained, and the AC particles worked as scaffolding to strengthen the slight biomolecules, thus expanding the active sites and effectively reducing the dissolution of the active materials. The pseudocapacitance fading mechanism was investigated by ex situ FTIR measurement and the porous structure ensures that the composite electrode has an enhanced specific capacitance of $248 \mathrm{~F} \mathrm{~g}^{-1}$ compared to 172.8 and $62.5 \mathrm{~F} \mathrm{~g}^{-1}$ for the respective AC and juglone samples. Besides, the excellent cyclic stability (retained $75 \%$ after 3000 charge-discharge cycles) was demonstrated. The highest area-specific capacitance of the composites was $1300 \mathrm{mF} \mathrm{cm}{ }^{-2}$. An asymmetric supercapacitor based on this composite electrode was assembled with an AC electrode as the counter electrode and exhibited good cyclic performance at a voltage of $1.2 \mathrm{~V}$ (retained $77 \%$ after 3000 charge-discharge cycles), which provides a high energy density of $12 \mathrm{~W} \mathrm{~h} \mathrm{~kg}^{-1}$ at a power density of $0.18 \mathrm{~kW} \mathrm{~kg}^{-1}$ and a high power density of $2 \mathrm{~kW} \mathrm{~kg}^{-1}$ at an energy density of $9 \mathrm{~W} \mathrm{~h} \mathrm{~kg}^{-1}$. This work explores the application of biomolecule-based composites in energy

storage devices and provides a potential strategy for constructing environmentally friendly electrodes.
\end{abstract}

Received 28th July 2019

Accepted 20th September 2019

DOI: $10.1039 / c 9 r a 05858 c$

rsc.li/rsc-advances

\section{Introduction}

With the improvements in productivity and economic prosperity, the consumption of energy is also rapidly increasing. ${ }^{1,2}$ In order to handle the crisis, renewable energy represented by solar energy and wind energy has been developed. However, limited by the unstable output and intermittent power supply of solar and wind, the efficient storage and use of new energy resources has become the critical factor to facilitate their practical application..$^{3-5}$ Supercapacitors, which can store and deliver electronic power quickly and reliably, have become promising energy storage devices for new energy resources. ${ }^{6}$ As the key component for supercapacitors, pseudocapacitance materials such as metallic

\footnotetext{
${ }^{a}$ State Key Laboratory of Electronic Thin Films and Integrated Devices, School of Optoelectronic Science and Engineering, University of Electronic Science and Technology of China, No. 4, Section 2, North Jianshe Road, Chengdu 610054, P. R. China.E-mail: jianhuaxu8023@126.com

${ }^{b}$ Engineering Research Center of Electronic Information Technology and Application, School of Electrical and Electronic Engineering, Chongqing University of Arts and Sciences, Chongqing 402160, P. R. China

$\dagger$ Electronic supplementary information (ESI) available. See DOI: $10.1039 / \mathrm{c} 9 \mathrm{ra} 05858 \mathrm{c}$
}

oxides and sulfides $\left(\mathrm{Co}_{3} \mathrm{O}_{4}, \mathrm{NiO}, \mathrm{CoS}, \mathrm{NiS}\right)$, with high specific capacitance, have been widely investigated. ${ }^{7-12}$ To meet the needs of clean and sustainable development, the investigation of naturally abundant and renewable materials with high electrochemical performance for new-generation green supercapacitors is intensively desired., ${ }^{\mathbf{4 , 1 3 , 1 4}}$ The limited reserves and reutilization of mineral resources for traditional electrode materials can hardly satisfy the booming demand and subsequent production quantities for energy storage devices, and will inevitably lead to rising prices. ${ }^{5,15,16}$ Besides, the non-biodegradability of current devices at the end of their working life will generate a large amount of e-waste with potential safety hazards. Therefore, safe, biodegradable and environmentally friendly biomaterials will occupy an important position in next-generation power sources. ${ }^{\mathbf{1 4 1} 17}$

Recently, inspired by some redox-active molecules acting as redox cofactors or electron shuttles within cells, renewablebiomass-based electrode materials with active functional groups, such as carbonyl, quinone and pteridines, have been shown to be promising electrode material candidates owing to their high electrochemical activity, environmental friendliness and affluent resources. ${ }^{18-20}$ Researchers have made tremendous 
progress in the fabrication of faradaic-type active electrode materials from renewable biomass. ${ }^{21-29}$ For example, through galvanostatic polymerization, Chen et al. fabricated a flexible integrated electrical cable using juglone/PPY with carbon fiber that exhibited an excellent performance of $0.72 \mathrm{mF} \mathrm{cm}^{-1}$, and $95 \%$ of the initial capacitance was retained after 1000 cycles. ${ }^{16}$ Furthermore, Yang et al. successfully fabricated a transitionmetal-free asymmetric supercapacitor (ASC) with conjugated lawsone/Ppy @carbon fibers as the faradaic-type negative electrode based on the coelectrodeposition of lawsone and pyrrole on carbon cloths; their ASC possessed a high volume energy density of $1.2 \mathrm{~mW} \mathrm{~h} \mathrm{~cm}^{-3} \cdot{ }^{30}$ The potential of using biomass as a supercapacitor electrode material has been demonstrated, while facile and large-scale processing methods are still needed for the practical application. However, as organic materials, biomass-based electrodes are subject to capacity fading owing to their poor conductivity and dissolution into the electrolytes, so a lot of effort still needs to be made to promote their practical application in energy storage devices.

Herein, we composited commercial AC with renewable juglone extracted from waste walnut peel as an electrode material using a simple but effective method. As expected, the composite electrode combined the advantages of both components. Through the research of electrodes with different mass ratios, the composite electrode with appropriate proportions revealed enhanced electrochemical performance, which not only came from the supporting role of AC particles but also resulted from the hierarchical and loose structure for highefficiency ion transfer paths during reactions. The electrochemical performance of the composite electrode was characterized in detail and related mechanisms were also discussed. Moreover, a coin-type asymmetrical supercapacitor was assembled using this composite material as the negative electrode and AC as the positive electrode.

\section{Experimental}

\subsection{Preparation of juglone-based electrodes}

The synthesis process for the juglone/AC composite is shown in Fig. 1. The extraction of juglone powder was based on an ultrasound-assisted extraction method. Firstly, the walnut peel was dried at $40{ }^{\circ} \mathrm{C}$ for several days and ground into a powder. Then the walnut peel powder was dissolved in $90 \%$ ethanol for $12 \mathrm{~h}$. With subsequent sonication for $45 \mathrm{~min}$ at $300 \mathrm{~W}$ intensity and centrifugation for several times, the filtrate was evaporated to dryness and the remaining products were collected.

Secondly, in order to prepare the composite electrodes, different ratios of juglone to YP50F activated carbon (Kalury Co. Ltd, Japan) AC (0:1, $1: 9,1: 3,1: 1,3: 1,1: 0)$ were dissolved and dispersed in $\mathrm{N}, \mathrm{N}$-dimethylformamide (DMF) solution with subsequent sonication for $30 \mathrm{~min}$ at $300 \mathrm{~W}$ intensity and mechanical stirring for $3 \mathrm{~h}$, and then vacuum drying overnight at $60{ }^{\circ} \mathrm{C}$. Then, the active material, conductive agent and binder poly(vinylidene fluoride) (PVDF) (Arkema, France) at a ratio of $8: 1: 1$ (wt $\%$ ) were mixed and stirred for $12 \mathrm{~h}$. Finally, the mixture was spread on a carbon cloth current collector $(3 \times 1$

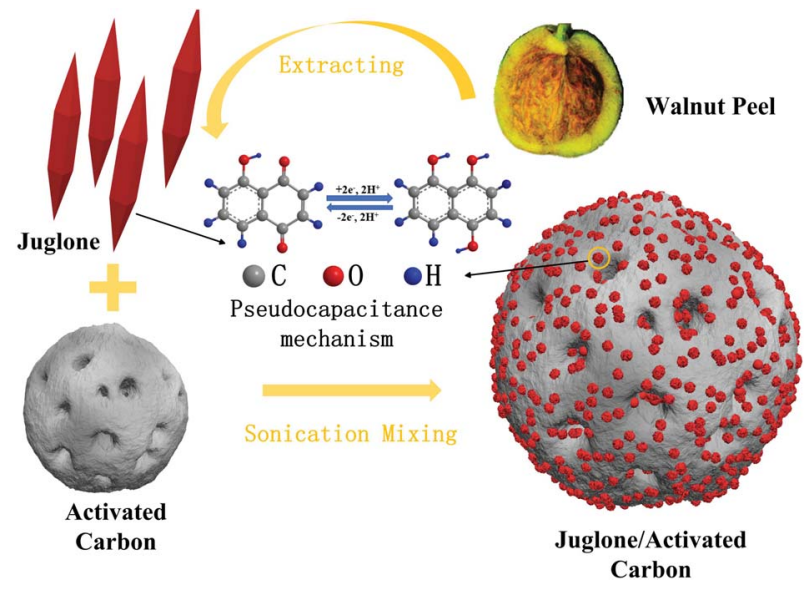

Fig. 1 Fabrication process for juglone/AC composite.

$\mathrm{cm}^{2}$ ) (CeTech Co Ltd, Taiwan in China) followed by vacuum

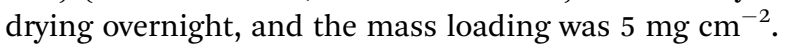

\subsection{Characterization}

The morphology of the prepared electrodes was characterized by scanning electron microscopy (SEM, type: S-4800, Hitachi). The analysis of chemical bonds was done using ex situ Fourier transform infrared spectroscopy (FTIR, type: 6700, Nicolet). The crystal structures were characterized by X-ray diffraction (XRD, PANalytical B.V. X'Pert Powder). The Brunauer-Emmett-Teller (BET) surface areas and pore distribution were characterized by $\mathrm{N}_{2}$ adsorption-desorption analysis (Micromeritics ASAP 2020 Xtended pressure sorption analyser).

\subsection{Electrochemical measurements}

The electrochemical properties of the resulting compounds were characterized by cyclic voltammetry $(\mathrm{CV})$, galvanostatic charge/discharge (GCD) and electrochemical impedance spectroscopy (EIS) measurements, carried out using a CHI660D electrochemical workstation ( $\mathrm{CH}$ Instruments). The $\mathrm{CV}$ measurements were taken in a standard three-electrode system with $1 \mathrm{M} \mathrm{H}_{2} \mathrm{SO}_{4}$ aqueous solution as the electrolyte, samplecoated carbon cloth as the working electrode, Pt foil as the counter electrode, and a saturated $\mathrm{Ag} / \mathrm{AgCl}$ electrode as the reference electrode, within the voltage range from -0.2 to $0.8 \mathrm{~V}$ and with scan rates from 1 to $20 \mathrm{mV} \mathrm{s}^{-1}$. EIS measurements were performed at an open circuit voltage over the frequency range of $0.1 \mathrm{~Hz}$ to $10^{5} \mathrm{~Hz}$ with a $5 \mathrm{mV}$ amplitude.

Asymmetric supercapacitors were fabricated to evaluate the electrochemical performance, which consisted of the juglone/ AC hybridized electrode (1:3 group) as the negative electrode, an $\mathrm{AC}$ electrode as the positive electrode, $1 \mathrm{M} \mathrm{H}_{2} \mathrm{SO}_{4}$ as the electrolyte, cellulose as the separator and a CR2032 coin-type cell stainless steel shell as the package. The as-prepared electrodes were cut into pieces with diameter of $1.4 \mathrm{~cm}$ and then assembled into cells in a $\mathrm{N}_{2}$-filled glove box. The CV tests were performed on a CHI660D electrochemical workstation with different scan rates in the voltage range of 0 to $1.2 \mathrm{~V}$. The 
charge/discharge tests were carried out on a CT 2001A Land battery testing system (Wuhan Land Electronics Co. Ltd, China) at different current densities in the potential range of 0-1.2 $\mathrm{V}$.

\subsection{Calculation of capacitance}

Considering that the quasi-reversible faradaic reactions in the electrodes make the galvanostatic charge-discharge curves nonlinear, the specific capacitance was calculated through the integral method: ${ }^{31}$

$$
\begin{gathered}
C_{\mathrm{m}}=\frac{2 i_{\mathrm{m}} \int V \mathrm{~d} t}{\left.V^{2}\right|_{V_{\mathrm{i}}} ^{V_{\mathrm{f}}}} \\
C_{\mathrm{A}}=\frac{2 i_{\mathrm{A}} \int V \mathrm{~d} t}{\left.V^{2}\right|_{V_{\mathrm{i}}} ^{V_{\mathrm{f}}}}
\end{gathered}
$$

Herein, $C_{\mathrm{m}}, C_{\mathrm{A}}\left(\mathrm{F} \mathrm{g}^{-1}, \mathrm{~F} \mathrm{~cm}^{-2}\right)$ represents the mass and areaspecific capacitance of GCD curves. $i_{\mathrm{m}}=I / m\left(\mathrm{~A} \mathrm{~g}^{-1}\right)$ and $i_{\mathrm{A}}=$ $I / A\left(\mathrm{~A} \mathrm{~cm}^{-1}\right)$ are the current density, where $I$ is the current and $m$ is the active mass of the electrodes (that is, effective mass of juglone and activated carbon). $\int V \mathrm{~d} t$ is the integral current area, where $V$ is the potential with initial and final values of $V_{\mathrm{i}}$ and $V_{\mathrm{f}}$, respectively, and $t(\mathrm{~s})$ is the discharging time.

The average energy density $E\left(\mathrm{~W} \mathrm{~h} \mathrm{~kg}^{-1}\right)$ and power density $\left(\mathrm{kW} \mathrm{kg}^{-1}\right)$ based on the total mass of the anode and cathode materials were calculated through the following formulas: ${ }^{31-33}$

$$
\begin{gathered}
E=\frac{1}{2} C_{\mathrm{m}} V^{2} \\
P=3600 \frac{E}{t}
\end{gathered}
$$

Herein, $C_{\mathrm{m}}$ represents the specific capacitance $\left(\mathrm{F} \mathrm{g}^{-1}\right) . V$ is the operation potential $(\mathrm{V})$ of the cell's integral current area.

\section{Results and discussion}

\subsection{Structure and morphology}

As the SEM images show, the juglone particles of the pure juglone sample form micro prisms and stack closely (Fig. 2c), which restrains the full contact between the electrolyte and the active sites. For the composite electrode (Fig. 2b), the activated carbon adsorbs the juglone molecules to form a rough nanostructure. The existence of AC greatly changed the morphology of juglone and significantly decreased the size of the juglone particles. Through the hybridization with AC with high specific surface area and strong adsorption ability, the juglone wrapped on the surface of the AC evenly and transformed to loose clusters with nanostructure. Moreover, the formation of a loose and porous film is favourable for inhibiting the dissolution of juglone molecules into the electrolyte. Full contact between the electrode surfaces and the electrolyte can effectively reduce the ion diffusion resistance and increase the number of active sites, thus improving the reversibility of the electrodes. However, as the amount of juglone increased, the aggregation of juglone particles increased (Fig. S1 $\dagger$ ). The promotion of the BET surface area of the $1: 9$ and $1: 3$ samples, compared with the pure juglone sample, is demonstrated by the $\mathrm{N}_{2}$ adsorption-
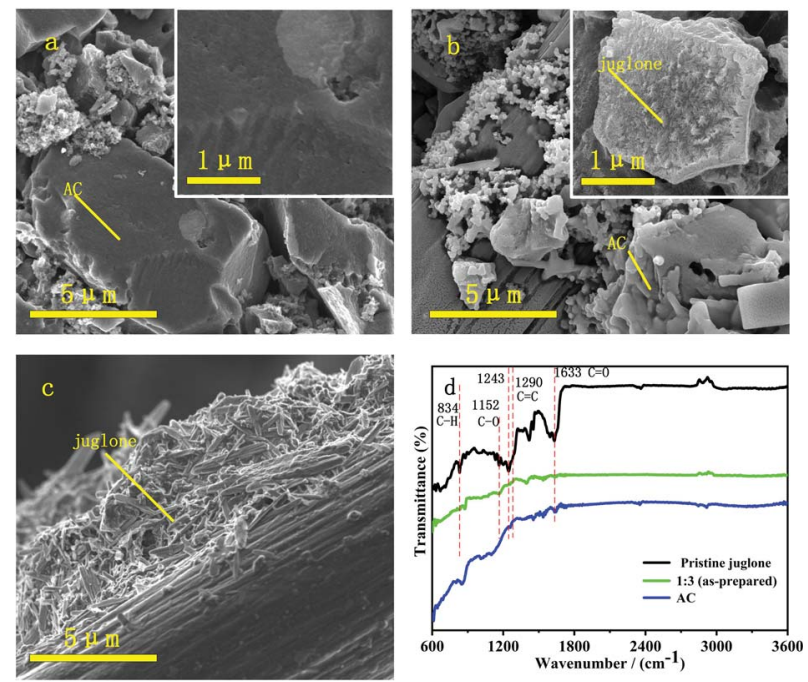

Fig. 2 SEM images of pure AC electrodes (a), $1: 3$ group (b) and pure juglone electrodes (c). (d) FTIR spectra for as-prepared electrodes.

desorption results in Fig. $\mathrm{S} 2 \mathrm{a}, \uparrow$ which shows BET surface areas of 1467,1055 , and $7.1 \mathrm{~m}^{2} \mathrm{~g}^{-1}$, respectively. However, when the mass ratio of juglone to AC was increased to $1: 1$ and $3: 1$, the BET surface area decreased to 316 and $26 \mathrm{~m}^{2} \mathrm{~g}^{-1}$, which might prohibit the fast redox reaction. The average adsorption pore diameters of the AC, $1: 9,1: 3,1: 1,3: 1$, and juglone samples are $2.41,2.29,2.31,2.68,9.59$ and $12.1 \mathrm{~nm}$, respectively. The $1: 3$ and $1: 9$ samples might exhibit better electrochemical performance for the surface characteristics.

Furthermore, the Fourier transform IR (FTIR) spectra (Fig. 2d) were analysed to verify the components and structures of the synthesized samples. Compared to the AC samples, the appearance of a new peak at $1633 \mathrm{~cm}^{-1}$ corresponds to the vibrational modes of $\mathrm{C}=\mathrm{O}$, and the peaks of the monomer at the wavenumbers of $834,1210(1152)$ and $1290 \mathrm{~cm}^{-1}$ are related to the $\mathrm{C}-\mathrm{H}$ bending, $\mathrm{C}-\mathrm{O}$ stretching vibration and $\mathrm{C}=\mathrm{C}$ stretching, respectively, on the benzene ring. ${ }^{34}$ The XRD patterns of these samples represent the crystal structures (Fig. S2c $\dagger$ ) and the intensity of the juglone characteristic peaks increased as the ratio of juglone increased. ${ }^{29}$ The above results demonstrate the successful extraction of juglone and its compositing with AC.

\subsection{Electrochemical measurements}

The CV measurements were taken to determine the electrochemical kinetics of the electrodes. In the CV curves of the different samples (Fig. 3a), a pair of redox peaks with poor symmetry for the pure juglone electrode $(1: 0)$ was observed at $-0.05 \mathrm{~V}$ and $0.23 \mathrm{~V}$. The wider reduction peak is related to a double-electron redox process. For the juglone electrode, the faradaic pseudocapacitance accounts for the vast majority of the total capacitance, but the reversibility of the redox process is unsatisfactory. The main problem may be the dense morphology formed by agglomeration of juglone, leading to insufficient contact between the active material and the electrolyte during the redox process. As a result, the amount of 

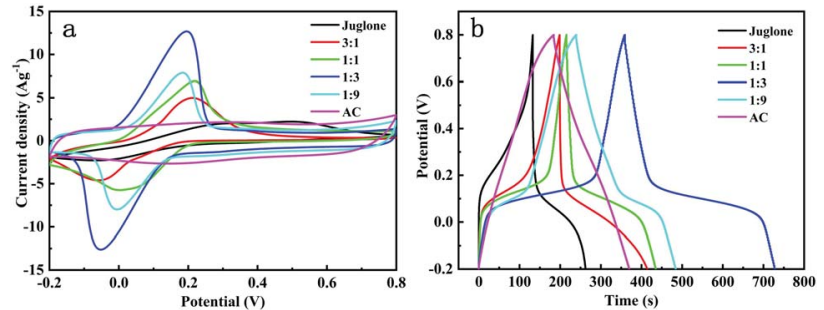

Fig. 3 (a) CV curves of AC electrode, juglone electrode and composite electrodes with different ratio of juglone (75 wt\%, $50 \mathrm{wt} \%, 25 \mathrm{wt} \%$, $10 \mathrm{wt} \%$ ) at a scan rate of $10 \mathrm{mV} \mathrm{s}^{-1}$ captured by a three-electrode system using $1 \mathrm{M} \mathrm{H}_{2} \mathrm{SO}_{4}$ as the electrolyte. (b) Galvanostatic chargedischarge plots under a current density of $1 \mathrm{~A} \mathrm{~g}^{-1}$.

active sites of juglone molecules available for the fast faradaic process decreases, causing the lowest specific capacitance among these samples. Furthermore, the reduction products may be more likely to dissolve in the electrolyte with the increased hydroxyl groups, resulting in irreversibility and capacitance loss.

As the mass ratio of juglone to AC gradually decreased from $3: 1$ to $1: 9$, the potential difference of the reduction and oxidation peaks decreased, which means the reversibility was effectively improved. Furthermore, the capacitance was significantly improved. In particular, when the ratio of juglone to AC was reduced to $1: 3$, the capacity and symmetry of the redox peaks appeared to be the best, which might be attributed to the smaller particle size of juglone, higher surface area of the electrode and higher juglone content (Fig. S1†). In the nearby area where the voltage is reversed, the current can quickly reach the plateau and the rectangular characteristic of the curves becomes more obvious. The specific capacitances are 172.8, 193.5, 248, 127.4, 91.1 and $62.5 \mathrm{~F} \mathrm{~g}^{-1}$ under $1 \mathrm{~A} \mathrm{~g}^{-1}$ for the $0: 1$ to $1: 0$ samples and the charge-discharge efficiency gradually increased to $50.5 \%, 65.9 \%, 89.4 \%$ and $78.8 \%$ in accordance with the trend of the CV curves (Fig. 3b). The total capacitance of the electrodes contains not only faradaic pseudocapacitance but also the contribution of the electrochemical double-layer capacitance. Through hybridizing with AC with a high specific surface area, the porous structure of the AC surface fully improves the utilization of the active sites and the ion mobility. However, when the percentage of juglone decreases to $10 \mathrm{wt} \%$, the capacity and polarization degree decrease distinctly, but they are still superior to those in the $1: 1$ group. The results show that the existing active sites are still effectively working but the lower content of juglone molecules will decrease the specific capacitance. As the AC content rises, the symmetry of the curves becomes better, and the charge-discharge curves contain an approximately linear area caused by the double-layer capacitance on the surface of the AC.

As the scan rates increases, the reduction peaks gradually move towards the negative potential (Fig. 4a). According to the calculated results, the peak current is proportional to the scan rate (Fig. 4c), which indicates that the redox reaction process is not limited by the semi-infinite linear diffusion and behaves like a capacitive process. It demonstrates that the faradaic
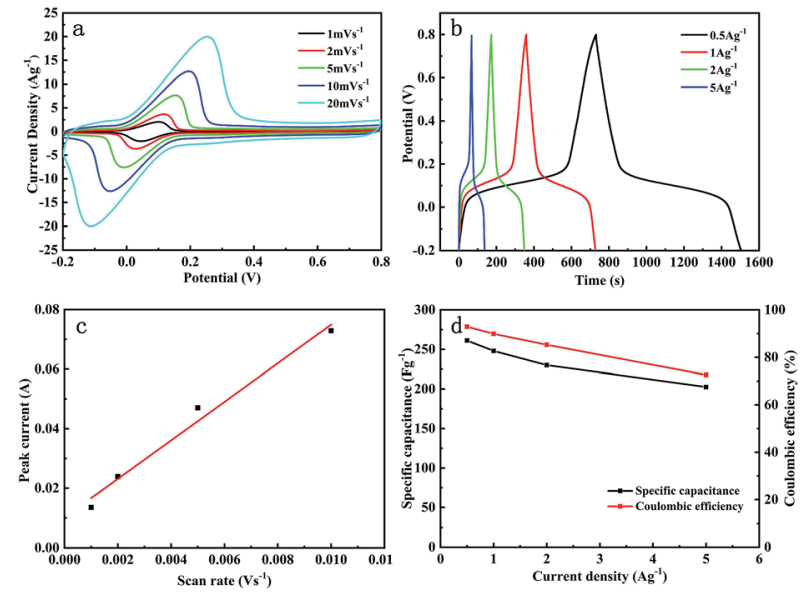

Fig. 4 (a) CV curves at different scan rates $\left(1,2,5,10,20 \mathrm{mV} \mathrm{s}^{-1}\right)$. (b) Galvanostatic charge-discharge plots under current densities of $0.5,1$, 2 , and $5 \mathrm{~A} \mathrm{~g}^{-1}$. (c) Relation curves between the oxidation peak currents and scan rates $(v)$. (d) Rate performance and coulombic efficiency of $1: 3$ samples.

energy storage mechanism of the composited electrode is mainly a capacitive pseudocapacitance process. The average discharge mass-specific capacitances of $265,248,230.1$, and $202.7 \mathrm{~F} \mathrm{~g}^{-1}$ and corresponding charge-discharge efficiencies of $89.4 \%, 87.2 \%, 84.6 \%$, and $73.2 \%$ were achieved at current densities of $0.5,1,2$ and $5 \mathrm{~A} \mathrm{~g}^{-1}$, respectively. The highest areaspecific capacitance was $1300 \mathrm{mF} \mathrm{cm}^{-2}$ with a current density of $2.5 \mathrm{~mA} \mathrm{~cm}{ }^{-2}$, which is close to that of a commercial supercapacitor electrode. The rate performance of the $25 \mathrm{wt} \%$ group was further investigated, as shown in Fig. 4d. For ideal polarizable electrodes, the specific capacitance is constant for charging/discharging current. Nevertheless, the specific capacitance and the coulombic efficiency of the as-prepared electrodes reduce when the discharge current density increases, which proves that the composite electrodes are non-ideal electrodes. This might be owing to the resistance caused by the porous structure and uneven distribution on the surface of the electrodes, so that many pores and active sites could not react in time under high current densities, thus making no contribution to the capacitance.

The cyclic performance measurements were performed at a scan rate of $0.01 \mathrm{~V} \mathrm{~s}^{-1}$ (Fig. 5b). The capacity retention of the juglone electrode was 33\%, but that of $25 \mathrm{wt} \%$ group was $75 \%$ after 3000 cycles owing to the porous structure and strong absorption effects of the AC particles. Simultaneously, the absorption signal at $1036 \mathrm{~cm}^{-1}$ originating from the vibrational modes of $\mathrm{C}=\mathrm{O}$ and a wider absorption peak for hydroxyl at $3343 \mathrm{~cm}^{-1}$ in the $25 \mathrm{wt} \%$ group (after GCD test) (Fig. 5c) indicate the introduction of new hydroxyl groups owing to the redox process of quinone carbonyls. ${ }^{35-37}$ The AC samples present the best cyclic performance for the non-faradaic EDLC process and the main reason for the AC capacitance fading might be the stripping from the current collector. However, the capacitance loss of juglone is caused by the faradaic pseudocapacitance process, which means the composites could not behave better 

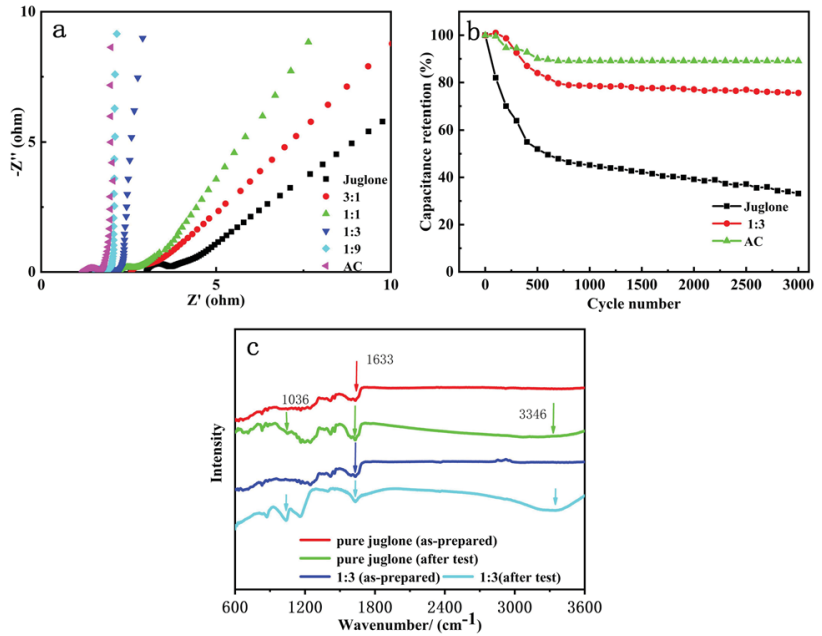

Fig. 5 (a) Nyquist plots of $A C$ impedance test measured between $0.1 \mathrm{~Hz}$ and $10^{5} \mathrm{~Hz}$ with an amplitude of $5 \mathrm{mV}$. (b) The cyclic capacity retention during 3000 cycles. (c) FTIR spectra for the electrode samples before and after the test.

than the pure AC in terms of the cyclic performance. Besides, the mass of the pure juglone electrode obviously decreases after the cyclic test, which might stem from dissolving in the electrolyte, leading to the capacitance fading. The masses of the composited samples do not change a lot, which might be owing to the strong adsorption of AC. The loss of capacitance might be mainly attributed to the irreversible component changing from $\mathrm{C}=\mathrm{O}$ to hydroxyl.

The electrochemical impedance spectroscopy (EIS) spectra of the composite electrodes shown in Fig. 5a also indicate the remarkably enhanced electrochemical reaction kinetics for these electrodes. The Nyquist plots for the juglone/AC electrodes show a decrease of the semicircle diameter in the highand middle-frequency regions compared to the pristine juglone electrode, indicating a faster electric response with much lower circuit resistance. Note that the oblique line representing diffusion with an angle of approximately $45^{\circ}$ from the $Z$-axis in the low-frequency region in the juglone electrode, which corresponds to the Warburg impedance, slopes towards the $Z^{\prime}$ axis gradually in the composite electrodes. As the content of juglone decreases, the diffusion coefficient increases, and the mass transfer environment and electrochemical reversibility of the electrode are enhanced with more pronounced capacitive characteristics. The significant improvement of the electrochemical performance from pure $\mathrm{AC}$ and juglone to the composites could be mainly rooted in three factors: (1) the reshaping of porous and nano-structural juglone particles for abundant pseudocapacitance active sites and a fast ion transfer path; (2) strong adsorption and adhesive force of juglone to AC, which enhances the electrochemical stability; and (3) good conductivity of AC to decrease the ESR of the whole composited electrodes.

Furthermore, in order to explore the performance under conditions closer to those for actual applications, CR2032-cointype asymmetrical supercapacitors were assembled with an AC
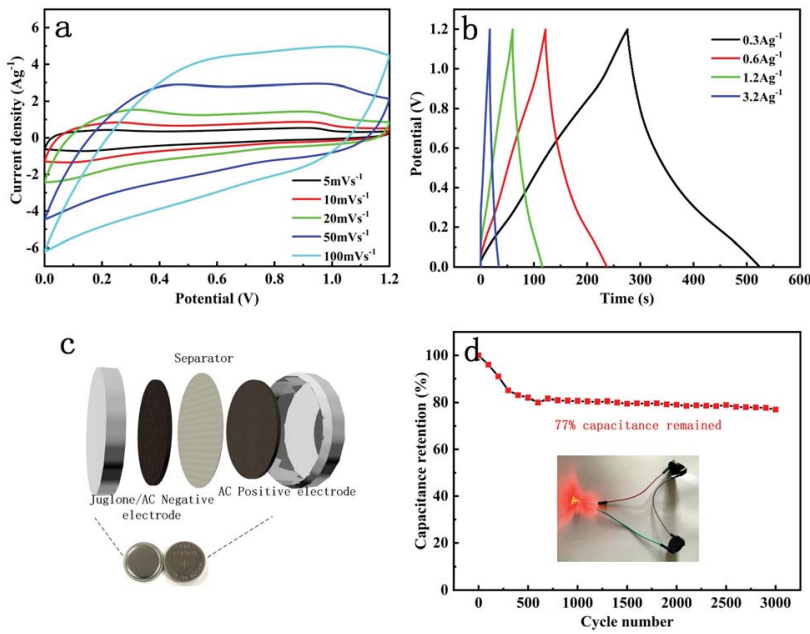

Fig. 6 (a) CV curves at different scan rates $\left(5 \mathrm{mV} \mathrm{s}^{-1}, 10 \mathrm{mV} \mathrm{s}^{-1}, 20 \mathrm{mV}\right.$ $\mathrm{s}^{-1}, 50 \mathrm{mV} \mathrm{s}^{-1}$, and $100 \mathrm{mV} \mathrm{s}{ }^{-1}$ ). (b) Charge/discharge plots under various currents ( $5 \mathrm{~mA}, 10 \mathrm{~mA}, 20 \mathrm{~mA}$, and $50 \mathrm{~mA}$ ). (c) Components and structure. (d) Cyclic performance of asymmetric supercapacitor.

electrode as the positive electrode and the electrochemical performance was further researched (Fig. 6c). The obtained galvanostatic charge-discharge curves are similar to triangular shapes, indicating good capacitive behaviour. There are two evident reduction peaks at $0.21 / 0.9 \mathrm{~V}$ at the scan rate of $5 \mathrm{mV} \mathrm{s}^{-1}$ related to the two-step reduction of juglone molecules. According to formulas (1), (2) and (3), the specific capacitance of the asymmetrical supercapacitor is $66,60,47.9,43.2 \mathrm{~F} \mathrm{~g}^{-1}$ at current densities of $0.3,0.6,1.2$ and $3.2 \mathrm{~A} \mathrm{~g}^{-1}$, respectively, and the highest energy density can reach $12 \mathrm{~W} \mathrm{~h} \mathrm{~kg}^{-1}$ at a power density of $0.18 \mathrm{~kW} \mathrm{~kg}^{-1}$ (highest power density of $2 \mathrm{~kW} \mathrm{~kg}^{-1}$ at energy density of $9 \mathrm{~W} \mathrm{~h} \mathrm{~kg}^{-1}$ ). Furthermore, $77 \%$ of the capacitance was retained after 3000 cycles at a scan rate of $0.01 \mathrm{~V} \mathrm{~s}^{-1}$, which proves the excellent stability of the asymmetric supercapacitor. An LED lamp could be lit up by two supercapacitors in series (embedded image in Fig. 6d). The electrochemical performance was comparable to many previously reported works (Table S1†). ${ }^{22,38-40}$

\section{Conclusions}

In summary, we have successfully prepared activated carbonsupported renewable juglone biomolecules as a pseudocapacitance electrode material in aqueous electrolyte via a facile and effective method. Among the different compositions, the electrode with a ratio of $1: 3$ presented the most impressive capacity performance. It exhibited the highest specific capacitance of $265 \mathrm{~F} \mathrm{~g}^{-1}$ at a current density of $0.5 \mathrm{~A} \mathrm{~g}^{-1}$ and maintained $76 \%$ of the initial capacitance when the current density was increased to $5 \mathrm{~A} \mathrm{~g}^{-1}$. It also has an improved cycle life with $75 \%$ capacitance retention after 3000 cycles. When asymmetric supercapacitors were assembled with an AC-based positive electrode, the composite electrodes demonstrated a remarkable energy density of up to $12 \mathrm{~W} \mathrm{~h} \mathrm{~kg}^{-1}$ (at $0.18 \mathrm{~kW} \mathrm{~kg}^{-1}$ ) and a power density of $2 \mathrm{~kW} \mathrm{~kg}^{-1}$ (at $9 \mathrm{~W} \mathrm{~h} \mathrm{~kg}^{-1}$ ). Besides, the device 
exhibited high stability with $77 \%$ retention of the initial specific capacitance after 3000 cycles. The incorporation with conductive AC particles significantly facilitates the electric conductivity of the composite electrodes and the charge transfer process during the redox reaction. Furthermore, the porous structure can relieve the volume change of the active materials during the charging/discharging cycle. The AC/juglone composite effectively reduced the dissolution of the active materials into the electrolyte and achieved improved cycling stability. Finally, compared to traditional transition-metal-based electrode materials, the faradaic-active carbon-organic composites satisfy the requirements of low cost and facile preparation process and may afford new inspiration for future energy storage.

\section{Conflicts of interest}

There are no conflicts to declare.

\section{Acknowledgements}

This work was supported by the National Natural Science Foundation of China (NSFC) (Grant No. 51477026, 61471085 \& 61774030), the National Science Funds for Creative Research Groups of China (Grant No. 61421002), Chongqing Postdoctoral Science Special Foundation (No. Xm2017051), and the Scientific and Technological Research Program of Chongqing Municipal Education Commission (No. KJ1601111).

\section{Notes and references}

1 J. G. Kim, D. P. Dubal and Y. Kim, Energy Technol., 2014, 2, 306.

2 G. Z. Chen, Prog. Nat. Sci.: Mater. Int., 2013, 23, 245-255.

3 B. Akinwolemiwa, C. Wei and G. Z. Chen, Electrochim. Acta, 2017, 247, 344-357.

4 C. P. Grey and J. M. Tarascon, Nat. Mater., 2016, 16, 45-56. 5 H. Gao, X. Wang, G. Wang, C. Hao, S. Zhou and C. Huang, Nanoscale, 2018, 10, 10190-10202.

6 S. Zhou, Z. Ye, S. Hu, C. Hao, X. Wang, C. Huang and F. Wu, Nanoscale, 2018, 10, 15771-15781.

7 M. Liu and J. Sun, J. Mater. Chem. A, 2014, 2, 12068-12074. 8 J. N. Marie-Francoise, H. Gualous, R. Outbib and A. Berthon, J. Power Sources, 2005, 143, 275-283.

9 Y. Shao, M. F. El-Kady, J. Sun, Y. Li, Q. Zhang, M. Zhu, H. Wang, B. Dunn and R. B. Kaner, Chem. Rev., 2018, 118, 9233-9280.

10 F. Wang, X. Wu, X. Yuan, Z. Liu, Y. Zhang, L. Fu, Y. Zhu, Q. Zhou, Y. Wu and W. Huang, Chem. Soc. Rev., 2017, 46, 6816-6854.

11 P. Simon and Y. Gogotsi, Nat. Mater., 2008, 7, 845-854.

12 S. Zhou, C. Hao, J. Wang, X. Wang and H. Gao, Chem. Eng. J., 2018, 351, 74-84.

13 D. H. Kang, M. Chen and O. A. Ogunseitan, Environ. Sci. Technol., 2013, 47, 5495-5503.

14 F. Xu, H. Xu, X. Chen, D. Wu, Y. Wu, H. Liu, C. Gu, R. Fu and D. Jiang, Angew. Chem., Int. Ed. Engl., 2015, 54, 6814-6818.
15 F. Wu, X. Wang, W. Zheng, H. Gao, C. Hao and C. Ge, Electrochim. Acta, 2017, 245, 685-695.

16 C. Huang, Y. Ding, C. Hao, S. Zhou, X. Wang, H. Gao, L. Zhu and J. Wu, Chem. Eng. J., 2019, 378, 122202.

17 H. Wang, Y. Yang and L. Guo, Adv. Energy Mater., 2017, 7, 1700663.

18 M. Armand, S. Grugeon, H. Vezin, S. Laruelle, P. Ribiere, P. Poizot and J. M. Tarascon, Nat. Mater., 2009, 8, 120-125.

19 K. Zhang, Z. Hu, Z. Tao and J. Chen, Sci. China Mater., 2014, $57,42-58$.

20 L. Guo, S. Dai, X. Sui and C. Chen, ACS Catal., 2015, 6, 428441.

21 V. Augustyn, P. Simon and B. Dunn, Energy Environ. Sci., 2014, 7, 1597.

22 H. Wang, F. Li, B. Zhu, L. Guo, Y. Yang, R. Hao, H. Wang, Y. Liu, W. Wang, X. Guo and X. Chen, Adv. Funct. Mater., 2016, 26, 3472-3479.

23 B. Genorio, K. Pirnat, R. Cerc-Korosec, R. Dominko and M. Gaberscek, Angew. Chem., Int. Ed. Engl., 2010, 49, 72227224.

24 J. Hong, M. Lee, B. Lee, D. H. Seo, C. B. Park and K. Kang, Nat. Commun., 2014, 5, 5335.

25 P. Hu, T. Chen, Y. Yang, H. Wang, Z. Luo, J. Yang, H. Fu and L. Guo, Nanoscale, 2017, 9, 1423-1427.

26 H. Wang, P. Hu, J. Yang, G. Gong, L. Guo and X. Chen, Adv. Mater., 2015, 27, 2348-2354.

27 Y. Sun, R. B. Sills, X. Hu, Z. W. Seh, X. Xiao, H. Xu, W. Luo, H. Jin, Y. Xin, T. Li, Z. Zhang, J. Zhou, W. Cai, Y. Huang and Y. Cui, Nano Lett., 2015, 15, 3899-3906.

28 M. Lee, J. Hong, H. Kim, H. D. Lim, S. B. Cho, K. Kang and C. B. Park, Adv. Mater., 2014, 26, 2558-2565.

29 L. Guo, A. Wang, P. Hu, A. Tian, R. Hao, D. Yu, J. Yang, D. Chen and H. Wang, RSC Adv., 2018, 8, 2077-2081.

30 Y. Yang, H. Wang, R. Hao and L. Guo, Small, 2016, 12, 46834689.

31 L. Q. Mai, A. Minhas-Khan, X. Tian, K. M. Hercule, Y. L. Zhao, X. Lin and X. Xu, Nat. Commun., 2013, 4, 2923.

32 X. Gao, W. Xing, J. Zhou, G. Wang, S. Zhuo, Z. Liu, Q. Xue and Z. Yan, Electrochim. Acta, 2014, 133, 459-466.

33 Y. Gao, W. Zhang, Q. Yue, B. Gao, Y. Sun, J. Kong and P. Zhao, J. Power Sources, 2014, 270, 403-410.

34 D. M. Yang, S. Y. Li, S. J. Li, J. Li, M. L. Sun and Y. Jin, For. Prod. J., 2009, 59, 79-82.

35 M. Park, D. S. Shin, J. Ryu, M. Choi, N. Park, S. Y. Hong and J. Cho, Adv. Mater., 2015, 27, 5141-5146.

36 S. Wang, L. Wang, K. Zhang, Z. Zhu, Z. Tao and J. Chen, Nano Lett., 2013, 13, 4404-4409.

37 S. Wang, L. Wang, Z. Zhu, Z. Hu, Q. Zhao and J. Chen, Angew. Chem., Int. Ed. Engl., 2014, 53, 5892-5896.

38 K. S. Lee, S. J. Kim, C. W. Park, I. Cho, P. J. H. Kim, V. G. Pol, I. Park and J. M. Ko, J. Ind. Eng. Chem., 2019, 78, 232-238.

39 G. S. Gudavalli, J. N. Turner and T. P. Dhakal, Nanotechnology, 2019, 30, 455701.

40 S. Sun, B. Ding, R. Liu and X. Wu, J. Alloys Compd., 2019, 803, 401-406. 